\title{
Fumarate/Ceramic Composite Based Scaffolds for Tissue Engineering: Evaluation of Hydrophylicity, Degradability, Toxicity and Biocompatibility
}

\author{
Juan Manuel Fernández ${ }^{1,2, *}$, María Susana Cortizo², and Ana María Cortizo ${ }^{1}$ \\ ${ }^{1}$ Laboratorio de Investigación en Osteopatías y Metabolismo Mineral (LIOMM), Facultad de Ciencias Exactas, \\ Departamento de Ciencias Biológicas, Universidad Nacional de La Plata, La Plata, Argentina \\ ${ }^{2}$ Facultad de Ciencias Exactas, Instituto de Investigaciones Fisicoquímicas Teóricas y Aplicadas (INIFTA), \\ Universidad Nacional de La Plata, CC 16 Suc. 4, La Plata, Argentina
}

\begin{abstract}
The present study was designed to investigate the possible cytotoxicity and biocompatibility of scaffolds based on previously characterized polymeric materials including poly- $\varepsilon$-caprolactone (PCL) or polydiisopropyl fumarate (blended or on their own), with or without hydroxyapatite (HAP). Water contact angle was also evaluated to determine the hydrophylicity of each scaffold. Degradation of different scaffolds was evaluated after a 10-week incubation in Dulbecco's modified eagle medium (DMEM) supplemented with 5\% (v/v) fetal bovine serum (FBS). Bone Marrow Stromal Cells (MSC) were grown on different scaffolds in an osteogenic medium, after which alkaline phosphatase activity (ALP) was evaluated. ALP activity increased when MSC were grown on PCL + HAP or Blend + HAP, as compared to PCL or Blend without HAP. The effect of different scaffolds on the proliferation of the macrophage cell line RAW 264.7, production of nitric oxide (NO) and secretion of pro-inflammatory cytokines was examined. After $72 \mathrm{~h}$, macrophages proliferated equally well on all scaffolds, maintaining a rounded morphology. None of the investigated scaffolds induced production of $\mathrm{NO}$ or cytokine release into the culture media, suggesting an absence of cytotoxicity. Therefore, these polymer- and HAP-based scaffolds could potentially be used as bone substitute materials.
\end{abstract}

Keywords: Cytotoxicity, Bone Tissue Engineering, Bone Marrow Stromal Cells, RAW 264.7 Macrophages, Poly-Epsilon-Caprolactone, Polydiisopropyl Fumarate.

\section{INTRODUCTION}

Medical procedures necessary for the treatment of significant bone tissue damage generate important costs to the health care system. ${ }^{1-5}$ Traditionally, in order to repair extensive lesions, patients have been submitted to either bone grafts (autografts, allografts and xenografts) or metallic grafts (prosthesis, osteosynthesis plates). Infectious disease transmission, lack of integration, fatigue and wear of metals, as well as the low availability of bone donors and graft rejection, represent some of the complications. Bone tissue engineering (BTE) uses concepts and principles from different fields (medicine, engineering, biochemistry) to restore or improve the damaged tissue. ${ }^{6}$ Three-dimensional scaffolds have been developed with various materials to support different types of cells and growth factors in its interior. In theory, once the scaffold is implanted in the patient, it will guide the regeneration

\footnotetext{
*Author to whom correspondence should be addressed.
}

of bone tissue. ${ }^{7}$ Currently, a number of different materials are being developed and evaluated for their possible application in the area of bone regeneration. ${ }^{7-11}$ However all these materials must meet several important requirements for their application in this area, such as: lack of immunogenicity, a timely biodegradation rate which allows for adequate growth of the new tissue, non-toxic degradation products, and appropriate mechanical properties in order to provide structural stability to a bone defect site while resilient to mechanical stress. ${ }^{12}$

We have previously reported the development, characterization and biocompatibility of a new compatibilized polymeric material based on two polymers, poly- $\varepsilon$-caprolactone (PCL) and polydiisopropyl fumarate (PDIPF). ${ }^{13}$ Using these homopolymers we have developed a blend (PCL-PDIPF) compatibilized by ultrasound, which shows better biocompatibility and mechanical properties compared to that of the homopolymers. We have also shown that the physicochemical and osteogenic properties 
improve with the addition of $1 \% \mathrm{w} / \mathrm{w}$ of hydroxyapatite (HAP) obtained and characterized in our laboratory from bovine bone. ${ }^{14}$ An important aspect in the development of a bone scaffold is the possible inflammatory response that the material could induce in an in vivo application. A first approach to this aspect may be reached by investigating the in vitro response of macrophages in culture, such as murine RAW 264.7 cells. This cell line is highly sensitive to cytotoxic agents and responds by sharply increasing pro-inflammatory cytokines such as interleukin-1 (IL-1) and tumor necrosis factor-alpha (TNF- $\alpha$ ) as well as the production of nitric oxide (NO). Due to these features, macrophages in culture constitute an excellent model for cytotoxicity studies with various substances in biological systems. ${ }^{15-22}$ In particular, studies that evaluate the possible cellular toxicity of biomaterials are of great interest for their possible biomedical applications. The main aim of this study was to evaluate the possible cytotoxicity and biocompatibility of scaffolds prepared with PCL, PCL + HAP (1\% w/w), PCL + PDIPF (blend), and blend + HAP (1\% w/w), using RAW 264.7 macrophages (cytotoxicity) or bone marrow stromal cells (MSC, for biocompatibility studies). In addition, the hydrophobicity and degradability of the materials were evaluated and correlated with cellular studies.

\section{MATERIALS AND METHODS}

\subsection{Preparation of Composite Scaffolds}

PCL was purchased from Aldrich and has a weightaverage molecular weight $\left(M_{W}\right)$ and polydispersity index (PI) of $65000 \mathrm{~g} \cdot \mathrm{mol}^{-1}$ and 1.4 , respectively, as indicated by the manufacturer. PDIPF was synthesized by microwave-assisted radical polymerization, using benzoyl peroxide as the initiator, as we have previously described. ${ }^{23}$ PDIPF weight-average molecular weight and polydispersity index were $131000 \mathrm{~g} \cdot \mathrm{mol}^{-1}$ and 2.0, respectively, as determined by size exclusion chromatography (SEC). Compatibilized blends of PCL and PDIPF (75:25 wt \%; Blend) were obtained by ultrasound using Bandelin HD60 equipment at $20^{\circ} \mathrm{C}$, following the previously described methodology. ${ }^{13}$ This particular composition was selected based on our preliminary studies. The $M_{w}$ and molecular weight distribution of the polymers were determined by size exclusion chromatography, using an LKB-2249 instrument at $25^{\circ} \mathrm{C}$. A series of $\mu$-Styragel columns, pore sizes $10^{5}, 10^{4}, 500$ and $100 \AA$, were used with chloroform as an eluent. The polymer concentration was $4-5 \mathrm{mg} / \mathrm{ml}$ and the flow rate was $0.5 \mathrm{ml} / \mathrm{min}$. The polymer was detected at $5.75 \mu \mathrm{m}$ with a Miran $1 \mathrm{~A}$ infrared spectrophotometer detector and calibration was performed using poly(methyl methacrylate) as a standard. Hydroxyapatite (HAP) was obtained from adult bovine femur as previously described. ${ }^{14}$ Composites of $1 \mathrm{wt} \%$ HAP and PCL or Blend were obtained by dissolution in chloroform and casting in a glass Petri dish. The solvent was allowed to evaporate at room temperature and then the resulting films were dried under vacuum until constant weight. The films were sterilized by UV exposure for $2 \mathrm{~h} .{ }^{13,14}$

\subsection{Characterization of Scaffolds}

\subsubsection{Water Contact Angle}

Water contact angle measurements of the films were carried out using a ramé-hart Model 500 goniometer (raméhart instrument co., USA) in the contact angle mode. Images were analyzed with DROPimage Advanced v2.2 software. All the tests were performed on the air-facing surfaces of the samples. Six measurements on different points were performed to calculate the mean static contact angle.

\subsubsection{Degradation of Films}

Film degradation was studied after incubation in DMEM supplemented with $10 \% \mathrm{v} / \mathrm{v}$ foetal bovine serum (FBS), in a humidified atmosphere of $95 \%$ air and $5 \% \mathrm{CO}_{2}$ at $37{ }^{\circ} \mathrm{C}$. Film samples were cut, weighed $\left(W_{0}\right)$ and placed in a 24-well plate. After UV sterilization, Dulbecco's modified eagle medium (DMEM) was added to each well. The culture medium was replaced once a week: during this weekly procedure, each film sample was washed, dried and weighed $\left(W_{t}\right)$. Film degradation was evaluated as the weight loss percentage $(\% \mathrm{~W})$, calculated according to the following Eq. (1)

$$
\% W=\left(W_{o}-W_{t}\right) \times 100 / W_{o}
$$

\subsection{Biocompatibility Studies with MSC}

Bone marrow stromal cells (MSC) were obtained from rats as described previous. (They were characterized by their ability to differentiate to various phenotype, such as osteoblasts, adipocytes, chondrocytes). ${ }^{24}$ Briefly, animals were sacrificed under anesthesia by rapid neck dislocation. MSC were collected by flushing the dissected femoral and tibial diaphysis medullary canal with DMEM (Invitrogen, Buenos Aires, Argentina) under sterile conditions. The resulting suspension was seeded in a $25 \mathrm{~cm}^{2}$ tissue culture flask. Cells were grown in DMEM supplemented with $5 \%(\mathrm{v} / \mathrm{v})$ FBS and antibiotics $(100 \mathrm{U} / \mathrm{ml}$ penicillin and $100 \mathrm{~g} / \mathrm{ml}$ streptomycin) in a humidified atmosphere of $95 \%$ air and $5 \% \mathrm{CO}_{2}$. For the experiments, polymeric films were cut to size and placed in a 24-well plate. MSC were plated on each film slice at a density of $5 \times 10^{4}$ cells/well, and cultured in $10 \%$ FBS-DMEM at $37{ }^{\circ} \mathrm{C}$. After cells reached confluence, they were induced to differentiate into osteoblasts using an osteogenic medium (DMEM$10 \%$ FBS containing $25 \mathrm{mg} / \mathrm{mL}$ ascorbic acid and $5 \mathrm{mM}$ sodium $\beta$-glycerol-phosphate), which was changed twice a week. Osteoblastic differentiation was evaluated by measuring alkaline phosphatase activity (ALP). After 15 days of osteogenic differentiation, cell monolayers were washed with phosphate-buffered saline (PBS) and the total cell 
extract was obtained with $200 \mathrm{uL} 0.1 \%$ Triton-X100. A $100 \mathrm{uL}$ aliquot of the extract was used to evaluate ALP by hydrolysis of $p$-nitrophenylphosphate ( $p$-NPP) into $p$ nitrophenol $(p-\mathrm{NP})$ at $37{ }^{\circ} \mathrm{C}$ for 1 hour. The absorbance of $p$-NP was recorded at $405 \mathrm{~nm}^{25}$. Aliquots of each cell extract were used for protein determination by Bradford's technique. ${ }^{26}$

Alternatively, the viability of MSC grown on scaffolds was estimated using a colorimetric tetrazolium assay. This assay measures the reduction of the tetrazolium salt 3-(4,5-dimrthylthiazol-2-yl)-2,5-diphenyl tetrazolium bromide (MTT) to formazan by intact mitochondria in living cells. Thus, absorbance change is directly proportional to the number of viable cells. ${ }^{27}$ For this assay, $3 \times 10^{4}$ MSC were plated in a 48 -multiwell plate (with a polymer film placed in each well) and cultured for 24 hours in $10 \%$ FBS-DMEM at $37{ }^{\circ} \mathrm{C}$. The medium was removed, cells were washed with PBS and fresh medium containing the MTT reagent (Sigma, USA) at a final concentration of $1 \mathrm{mg} / \mathrm{mL}$ was added. After a 3-hour incubation each film was removed, placed in a new 48-multiwell plate and washed again with PBS. Color was developed by addition of $200 \mu \mathrm{L}$ dimethylsulfoxide (DMSO) (Merck, Argentina) and mixing in a plate shaker for 20 minutes, after which absorbance was measured at $540 \mathrm{~nm} \cdot{ }^{27}$

\subsection{Cytotoxicity Studies with RAW 264.7 Macrophages}

Murine macrophage RAW 264.7 cells were grown in DMEM supplemented with 5\% (v/v) FBS and antibiotics (100 $\mathrm{U} / \mathrm{ml}$ penicillin and $100 \mathrm{~g} / \mathrm{ml}$ streptomycin) in a humidified atmosphere of $95 \%$ air and $5 \% \mathrm{CO}_{2}$. For the experiments, polymeric films were cut to size, inserted in a 24-well plate and macrophages plated on them.

A mitogenic bioassay was carried out as described by Okajima et al. ${ }^{28}$ with some modifications. Briefly, RAW 264.7 cells grown on the films were washed with PBS and fixed with $5 \%$ glutaraldehyde/PBS for $10 \mathrm{~min}$. The cells were then stained with $0.5 \%$ crystal violet ( $25 \%$ methanol) for $10 \mathrm{~min}$. Excess dye was discarded and the plate was washed with water and dried. The dye taken up by the cells was extracted using $0.5 \mathrm{~mL} /$ well $0.1 \mathrm{M}$ glycine/HCl buffer, $\mathrm{pH} 3.0 / 30 \%$ methanol, transferred to test tubes and the absorbance was read at $540 \mathrm{~nm}$. The correlation between cell number and absorbance at $540 \mathrm{~nm}$ has been previously established. ${ }^{29,30}$ Alternatively, cells were stained with hematoxylin-eosin (H-E) after 24 hours of culture for observation with an optical microscope.

Nitric oxide (NO) production was assessed using Griess' reaction $^{29,31}$ (using sulfanilic acid as the diazotizing agent and $N$-1-napthylethylene diamine as the coupling agent). The stable end-product of NO and nitrite released into the culture medium by RAW 264.7 cells was measured after 24,48 and 72 hours of culture. Briefly, $400 \mu \mathrm{l} \mathrm{sam-}$ ples of conditioned media or nitrite standards $0-100 \mathrm{nM}$ were mixed with $400 \mu$ l of Griess' reagent (1\% sulfanilamide and $0.1 \%$ naphthylethylene-diamine in 5\% phosphoric acid) and absorbance was measured at $530 \mathrm{~nm}$ against a blank prepared with non-conditioned medium.

The pro-inflammatory cytokines tumor necrosis factoralpha (TNF- $\alpha$ ) and interleukin-1 (IL-1) released into the culture medium by RAW 264.7 cells were evaluated at 24 , 48 and 72 hours by ELISA kits (Mouse IL- $1 \beta$ LISA Set, BD Biosciences OptEIA ${ }^{\mathrm{TM}}$ and TNF- $\alpha$ kits Mouse ELISA Set, BD Biosciences OptEIA ${ }^{\mathrm{TM}}$ ) following the manufacturers instructions, as we have previously described. ${ }^{32}$

For NO and pro-inflammatory cytokine production, RAW 264.7 cells were also plated on standard culture tissue dishes with or without lipopolysaccharide (LPS, $0.1 \mu \mathrm{g} / \mathrm{ml}$ ) as positive and negative controls, respectively.

\subsection{Statistical Analysis}

Student's $t$-test was used for comparisons between control and experimental groups. All results are expressed as mean \pm S.E.M. and represent at least three different experiments performed in triplicate.

\section{RESULTS AND DISCUSSION}

A large number of materials are currently being studied for their application in bone tissue engineering. However, there have been comparatively few studies on the possible cytotoxic effects of these materials on cultured cells, a relevant feature for biomedical applications. We have previously developed a new composite biomaterial based on a compatibilized PCL-PDIPF blend including $1 \% \mathrm{wt}$ HAP, and thoroughly studied its physicochemical, mechanical and biocompatibility properties using UMR106 rat osteosarcoma cells and MC3T3E1 mouse calvaria-derived cells. ${ }^{13,14}$ We have also shown that both PCL and PDIPF can be degraded by macrophages. Moreover, the studied polyfumarates, although possessing a $\mathrm{C}-\mathrm{C}$ main chain (see Scheme 1), can be degraded under in vitro cellular conditions by a phagocytic process. ${ }^{8}$ Cell behavior is not only dependent on the chemical nature of a scaffold but also on its topographical features, ${ }^{33,34}$ hydrophilicity ${ }^{34-38}$ and the initial cell-surface interaction. ${ }^{37-39}$ Many research groups have focused their studies on the interactions between cells and matrices in order to elucidate why membranes with hydrophobic groups can facilitate cell attachment to
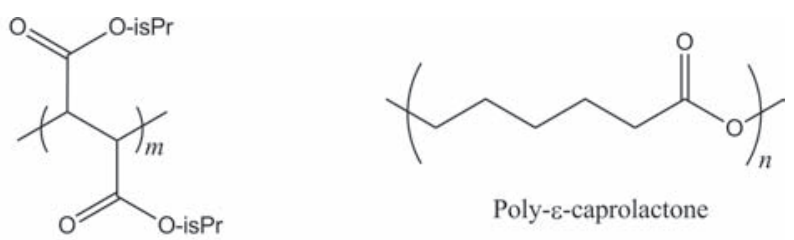

Polydiisopropyl fumarate

Scheme 1. Chemical structure of the polydiisopropyl fumarate and poly- $\varepsilon$-caprolactone used for scaffold preparation. 


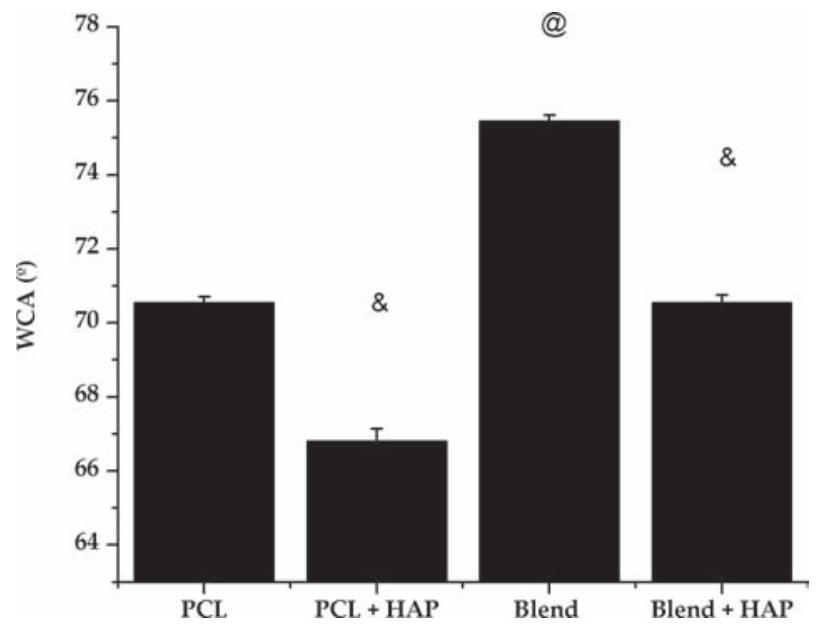

Fig. 1. Water contact angle (WCA) of PCL, PCL + HAP, Blend and Blend + HAP. PCL was more hydrophilic than the Blend, and the incorporation of HAP to the polymers increased their hydrophylicity. \&: $p<$ 0.001 PCL versus PCL+HAP and Blend-versus Blend+HAP; @: $p<$ 0.001 Blend versus PCL.

scaffolds, ${ }^{40-44}$ and have concluded that it is due to initial interactions between cells and scaffolds that are dependent on weak bonds such as dipole-dipole. Although it is still not understood how the hydrophobicity or hydrophilicity of materials can regulate cellular activity and tissue regeneration, this is an important parameter to consider when designing a scaffold. Thus, polymer-cell interactions can be related to the wetting properties of the film, thus influencing cellular behavior. ${ }^{45}$ In order to investigate these aspects, the water contact angle (WCA) of the membranes was evaluated in the present study. Figure 1 shows the WCA of PCL and compatibilized blend polymer membranes with and without the addition of HAP. The Blend exhibited a significantly higher WCA $\left(75^{\circ}\right)$ than the PCL membrane $\left(70^{\circ}\right)$. We have previously reported the WCA of a pure PDIPF film $\left(93^{\circ}\right),{ }^{8}$ thus our present observations for the blend are expected based on its chemical composition and are consistent with the chemical structure of the polymers. PDIPF is a polymer comprising a carboncarbon chain that supports pendent isopropyl ester groups, a structural feature that confers greater hydrophobicity than the polyester PCL. On the other hand, the incorporation of HAP to both PCL and Blend scaffolds significantly reduced the WCA ( $66^{\circ}$ and $70.5^{\circ}$, respectively). These observations are in agreement with previous reports in which HAP was included in different scaffolds. ${ }^{34,46}$ In our present study, the order of hydrophobicity of our membranes was of PCL-HAP $<$ PCL $<$ Blend-HAP $<$ Blend. These differences could not be important from the point of view of cell growth. We have previously reported a change in the surface rough of membranes with or without HAP. ${ }^{14}$ This property could in fact be expected to modulate the adhesion, proliferation and differentiation of cells.

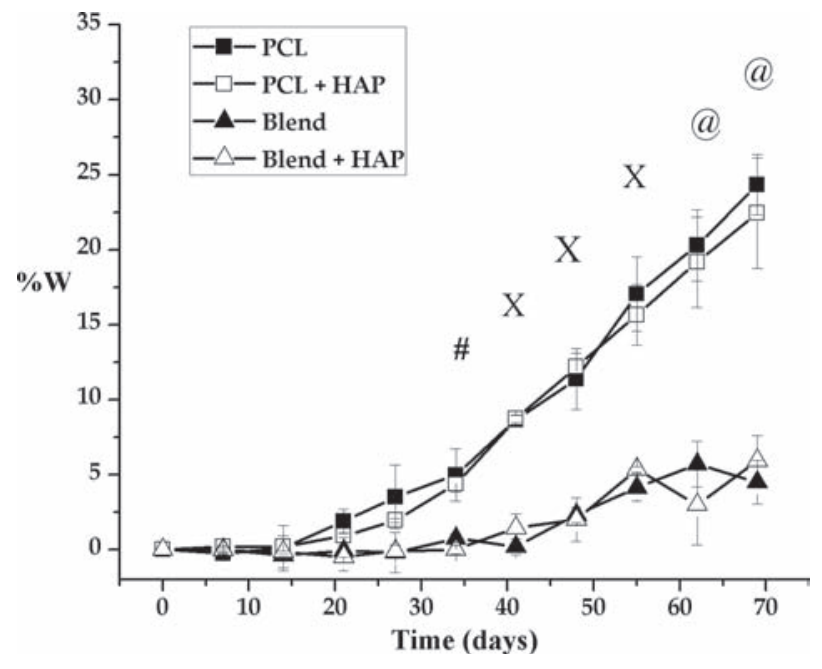

Fig. 2. Degradation of PCL, PCL + HAP, Blend and Blend + HAP in DMEM $+10 \%$ FBS. PCL was degraded faster than the Blend after 35 days. Incorporation of HAP did not modify the degradation rate of polymers. \#: $p<0.05 ; X: p<0.01 ; @: p<0.001$.

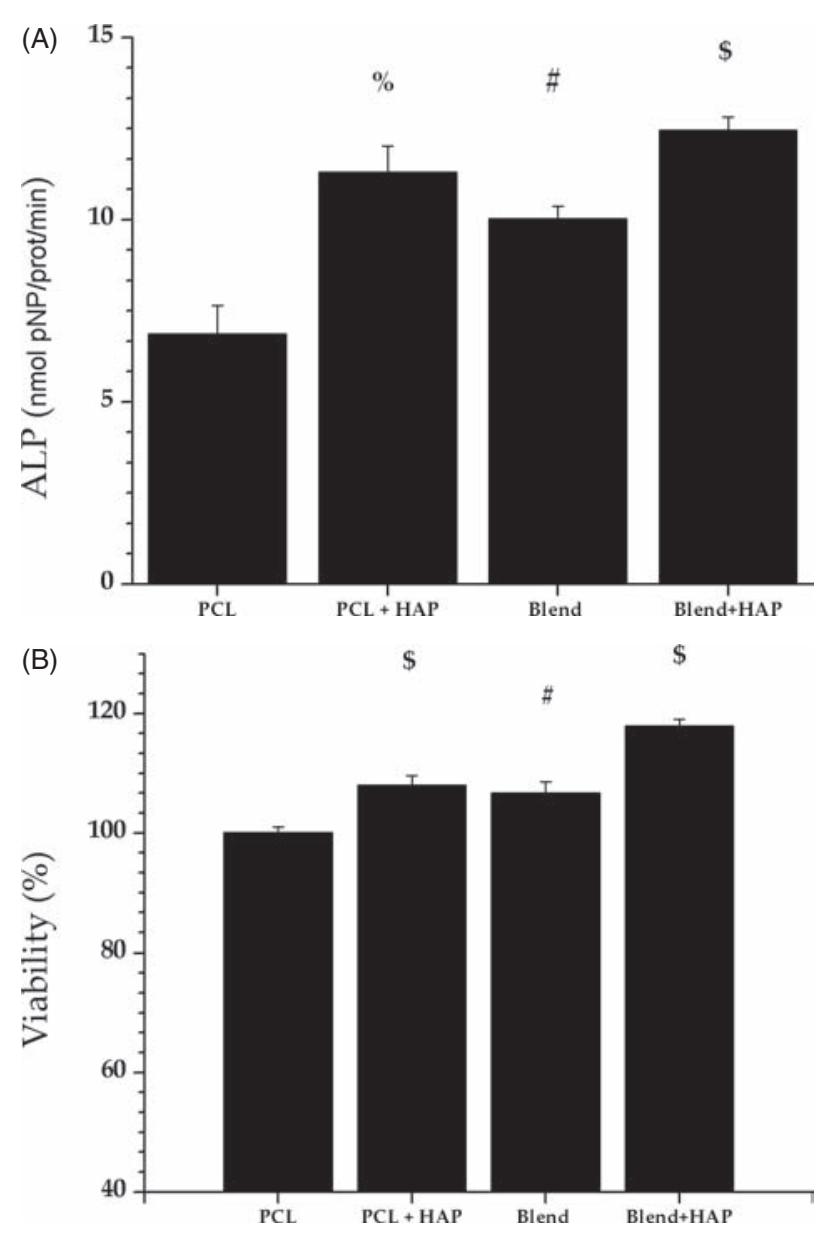

Fig. 3. Biocompatibility studies. (A) Osteogenic differentiation of rat MSC was evaluated by ALP activity. $\%: p<0.01$ versus PCL. $\$: p<$ 0.05 versus Blend; \#: $p<0.05$ PCL versus Blend. (B) Viability of MSC cultured on films (shown as \% of PCL). \#: $p<0.05$ PCL versus Blend. $\$: p<0.05$ versus polymers without HAP. 


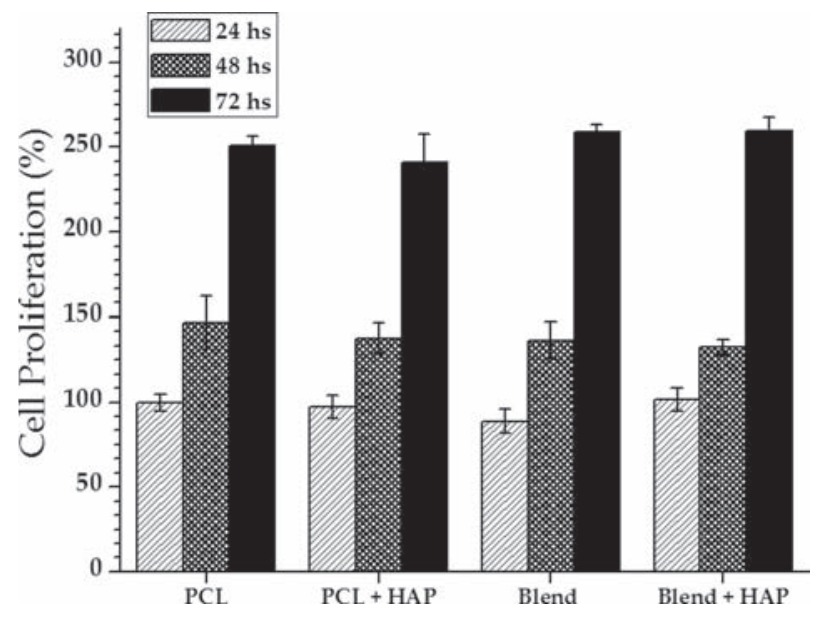

Fig. 4. Proliferation of RAW 264.7 macrophages. RAW 264.7 cultured for 24 hours on PCL, PCL + HAP, Blend or Blend + HAP (shown as \% of PCL). Macrophages proliferated equally on all the polymers for the periods of time evaluated.

Another aspect that must be considered is that materials must possess an adequate degradation rate, to ensure that the scaffold does not collapse in the time-span needed for cells to regenerate tissue. ${ }^{9,47-49}$ We evaluated the degradation rate of the different films incubated in DMEM/5\% FBS for 10 weeks (Fig. 2). Although initially (0-20 days), no significant degradation of the scaffolds was observed, after 5 weeks both PCL and PCL + HAP scaffolds showed a faster degradation (almost twice) than either Blend or Blend + HAP scaffolds. These differences could be due to the chemical nature of the polymers. The blend includes PFIP, a polymer with a $\mathrm{C}-\mathrm{C}$ catenarian structure that is more difficult to degrade than PCL. The latter is more susceptible to hydrolytic degradation due to its ester bonds $[-\mathrm{C}-\mathrm{O}-(\mathrm{C}=\mathrm{O})-] .{ }^{50}$ Interestingly, the introduction of HAP into the scaffold did not modify the degradation rate. Many authors have studied the degradation of polymeric materials under different conditions. For example, Huang et al. ${ }^{51}$ studied the degradation of a PLA-PCL-PLA triblock polymer in phosphate buffer solution (PBS). Other authors have used different enzyme solutions such as lipase $^{52}$ or proteinase, ${ }^{53}$ as well as in the presence of microorganisms ${ }^{54}$ to determine the degradation kinetics of materials. We used DMEM + FBS because we believe this medium is more representative of physiological conditions than with lipase or PBS. Our results are in agreement with those of other researchers ${ }^{51,54}$ that have shown a slow hydrolytic degradation for PCL when compared with other polyesters, but faster than that of polymers such as PDIPF (which is included in our compatibilized blend). We believe that the relatively slow degradation rate of PCL or the PCL-PDIPF blend, would allow MSC to proliferate, differentiate into osteogenic cells and thus regenerate the damaged bone tissue.

We next evaluated whether the PCL or PCL-PDIPF blend polymers with and without hydroxyapatite (HAP), were cytotoxic for RAW 264.7 macrophages and/or biocompatible with MSC.

The ability of MSC to differentiate into osteoblasts and express ALP when grown on different films was investigated after 15 days of osteogenic induction (Fig. 3(A)). ALP expression was higher in cells growing on the Blend than in cells cultured on PCL films (\#: $p<0.05)$. The addition of HAP to films increased cellular expression of ALP both for PCL (\%: $p<0.01)$ and for the Blend (\$: $p<0.05)$. We have previously demonstrated that the incorporation of HAP to PCL and Blend, increased their biocompatibility with cloned osteoblastic cell lines. ${ }^{14}$ In the present study, we have used MSC to evaluate biocompatibility, since these cells represent a better model for bone tissue regeneration. The viability of MSC grown on different films was evaluated with the MTT assay. Figure 3(B) shows that the viability of cells was higher for the Blend than for PCL (\#: $p<0.05)$, and that the addition of HAP to polymers further increased cellular viability. We also measured WCA to evaluate the hydrophobicity of the different scaffolds, and found that the addition of HAP to the polymers decreased their WCA. This effect was associated with an increase in the viability of MSC. Another interesting approach could be to study to differentiation without using an osteogenic medium, but get it as a consequence

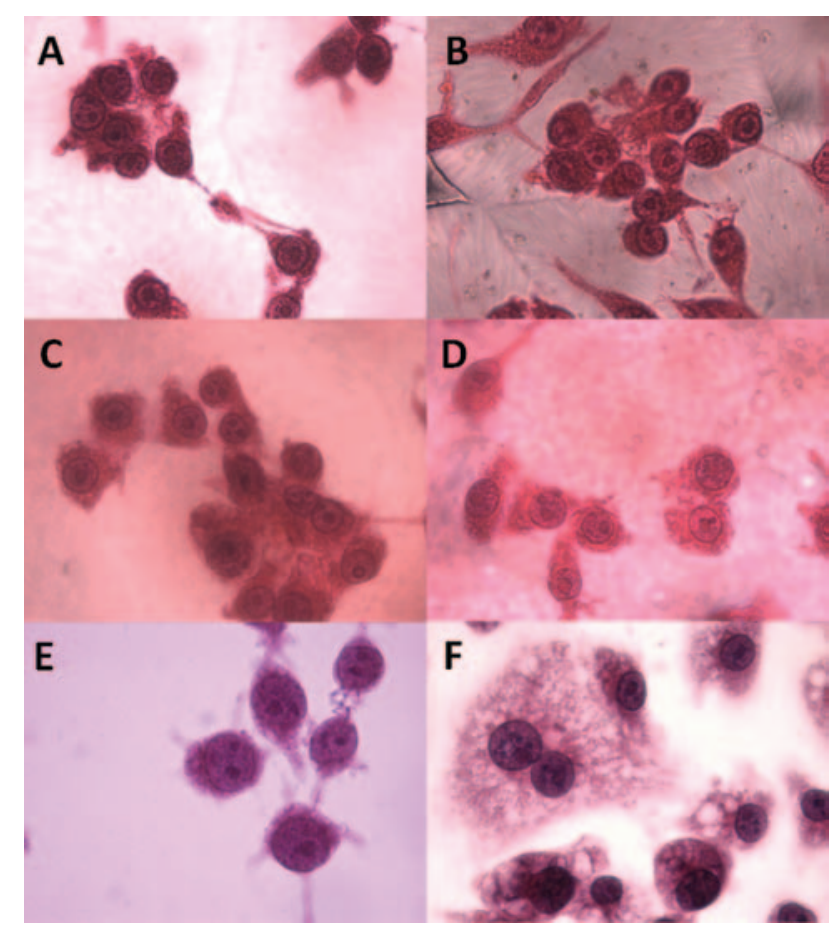

Fig. 5. Morphology of RAW 264.7 macrophages. Cells stained with $\mathrm{H}-$ E after 24 hours of culture on PCL (A), PCL + HAP (B), Blend (C), Blend + HAP (D), standard plastic tissue culture dish (E) or plastic tissue culture dish in the presence of LPS (F). In the positive control (F), macrophages appear activated with multiple cellular extensions and a vacuolated cytoplasm; these characteristics are absent in macrophages grown on control and polymeric scaffolds. Magnification X1000. 

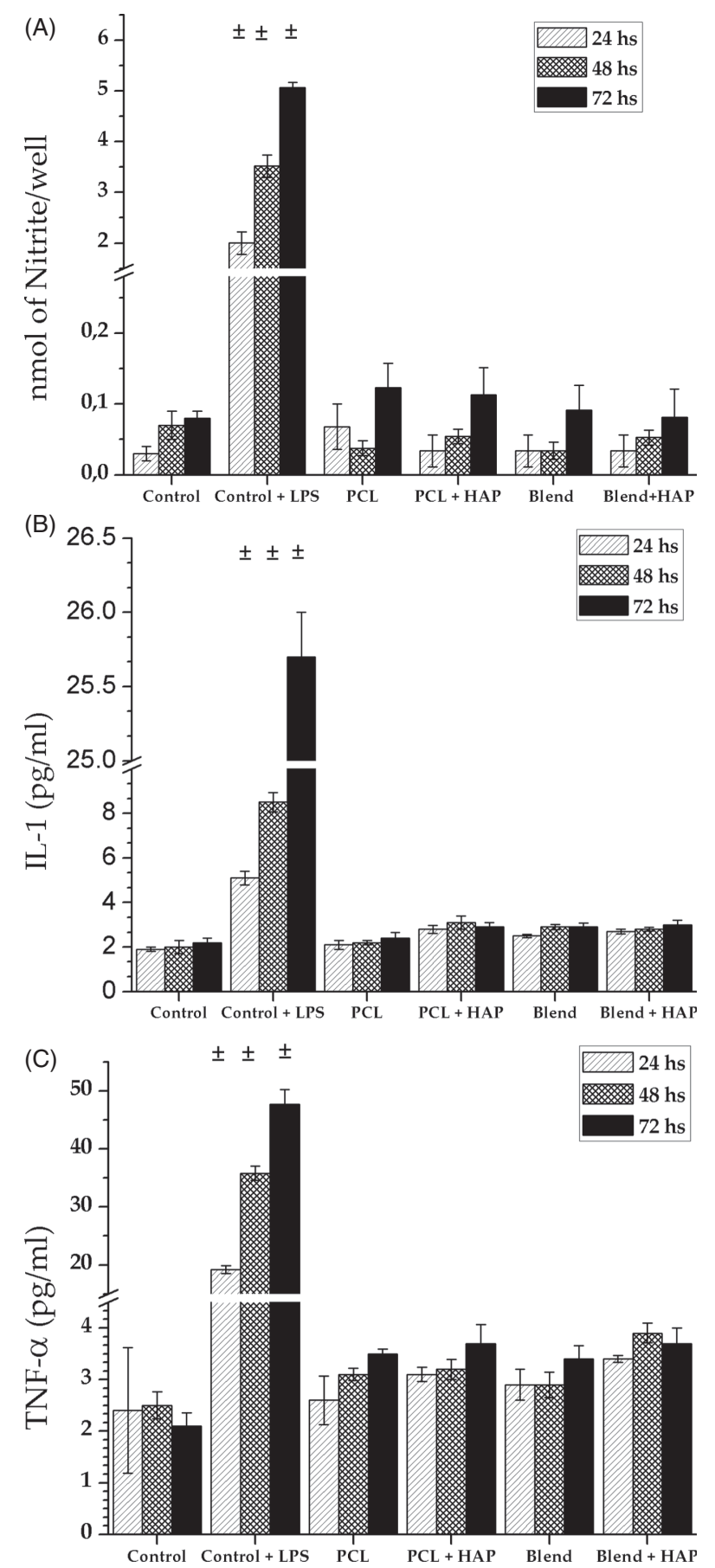

Fig. 6. cytotoxicity studies. Levels of NO (expressed as nmol of Nitrite/well) (A), IL-1 (B) and TNF- $\alpha$ (C) produced by RAW 264.7 macrophages cultured on standard plastic tissue culture dishes (control), control + LPS, PCL, PCL + HAP, Blend or Blend + HAP. No significant differences were observed in the release of NO or secretion of cytokines by macrophages grown either on control culture dishes or on the different polymeric scaffolds. LPS induced a significant increase in all proinflammatory markers. $\pm: p<0.001$.

of cues coming from the substrate. New experiments are in progress to test this hypothesis.

Previously, our group ${ }^{32}$ and other researchers ${ }^{16,18}$ have shown that macrophages are a very sensitive culture model to evaluate possible in vitro inflammatory responses to a material with potential applications in tissue engineering. In the present study, we investigated the capacity of RAW 264.7 macrophages to proliferate on different scaffolds. Figure 4 shows that after 24-72 hours, cells proliferate equally well on all scaffolds. Thus, the hydrophilicity (and/or chemical nature) of our membranes apparently does not affect the proliferation of macrophages, contrary to what has been reported for other cell lines. ${ }^{8,13,14,32,55}$ In addition, we analyzed cell morphology after H-E staining. Cells growing on different scaffolds or on standard plastic tissue culture dishes showed a rounded morphology with few cytoplasmic extensions (Fig. 5(A)). On the contrary, when cells on plastic dishes were exposed to LPS, as a positive control of cytotoxicity, they showed an expanded and vacuolated cytoplasm with several extensions, suggesting activation of the macrophages (Fig. 5(B)).

As has been pointed out above, a very important requirement of biomaterials is that the scaffolds must not be toxic because they have been designed for incorporation into a living organism. Therefore, the materials developed in our laboratory were evaluated to determine their potential cytotoxicity, assessing the release of NO and the pro-inflammatory cytokines TNF- $\alpha$ and IL- $1,{ }^{15-17,19-22}$ by macrophages cultured on the different materials for 2472 hours. Figure 6(A) shows that NO release from cells grown on all evaluated polymers was similar to control (standard plastic tissue culture dish). When $0.1 \mu \mathrm{g} \cdot \mathrm{ml}^{-1}$ LPS was added to the culture medium as a positive control of cytotoxicity, a statistically significant $( \pm: p<0.001)$ increase in NO release to the culture media was detected. In addition, Figures 6(B) and (C) shows that there was no significant difference in the levels of TNF- $\alpha$ and IL-1 secreted into the culture medium between cells grown on the different polymeric scaffolds, or standard plastic tissue culture dishes. Again, in the presence of LPS cells responded by increasing the production of both cytokines after 24 to $72 \mathrm{~h}$ of culture. Thus, the secretion levels of NO, TNF- $\alpha$ and IL- 1 to the culture media correlate with cell morphology (Figs. 5(A)-(D)), suggesting that the scaffolds do not induce cytotoxicity during the periods of time tested. On the other hand, macrophages responded as expected to an established toxic agent such as LPS by becoming morphologically (Fig. 5(F)) and functionally activated, increasing NO and cytokine release into the culture media.

\section{CONCLUSION}

Our in vitro results show that RAW 264.7 macrophages cultured on films of PCL and PCL-PDIPF Blend, with or without HAP, do not increase the secretion of NO, IL-1 or TNF- $\alpha$ during the evaluated culture periods. In addition, the differences in hydrophobicity (and/or chemical nature) between the different scaffolds do not induce changes in 
the proliferation of RAW 264.7 macrophages. However, changes in hydrophobicity (and/or chemical nature) can produce changes in the viability and biocompatibility of MSC. In addition, the degradation rate of PCL and PCLPDIPF Blend, with or without HAP was shown to be relatively slow in comparison with another polyesters scaffolds. Thus, these polymer-based scaffolds could be potentially useful for clinical applications such as materials for bone tissue engineering. In order to investigate this important issue, we are currently undertaking in vivo studies using these new materials.

Acknowledgments: This work was partially supported by grants from Facultad de Ciencias Exactas, Universidad Nacional de La Plata (UNLP), Comisión de Investigaciones Científicas de la Provincia de Buenos Aires (CICPBA) and Agencia Nacional de Promoción Científica y Tecnológica (PICT 1083 BID-1728/OC-AR). Juan Manuel Fernández is indebted to CONICET for the fellowship awarded. Ana María Cortizo is a member of the Carrera del Investigador Científico de la CICPBA.

\section{References and Notes}

1. M. Braddock, M. P. Houston, C. Campbell, and P. Ashcroft, Physiology 16, 208 (2001).

2. P. X. Ma, R. Zhang, G. Xiao, and R. Franceschi, Journal of Biomedical Materials Research 54, 284 (2001).

3. M. Morosano, A. Masoni, and A. Sánchez, Osteoporosis International 16, 1339 (2005)

4. J. R. Porter, T. T. Ruckh, and K. C. Popat, Biotechnology Progress 25, 1539 (2009)

5. L. Schurman, A. Bagur, H. Claus-Hermberg, O. D. Messina, A. Negri, and A. Sanchez, Revista Argentina de Osteología 6, 27 (2007).

6. R. Langer and J. Vacanti, Science 260, 920 (1993)

7. P. X. Ma, Materials Today 7, 30 (2004).

8. M. S. Cortizo, M. S. Molinuevo, and A. M. Cortizo, Journal of Tissue Engineering and Regenerative Medicine 2, 33 (2008).

9. D. W. Hutmacher, Biomaterials 21, 2529 (2000).

10. V. Karageorgiou and D. Kaplan, Biomaterials 26, 5474 (2005).

11. X. Liu and P. X. Ma, Annals of Biomedical Engineering 32, 477 (2004).

12. E. L. Hedberg, C. K. Shih, J. J. Lemoine, M. D. Timmer, M. A. K. Liebschner, J. A. Jansen, and A. G. Mikos, Biomaterials 26, 3215 (2005).

13. J. M. Fernandez, M. S. Molinuevo, A. M. Cortizo, A. D. McCarthy, and M. S. Cortizo, Journal of Biomaterials Science, Polymer Edition 21, 1297 (2010)

14. J. M. Fernandez, M. S. Molinuevo, M. S. Cortizo, and A. M. Cortizo, Journal of Tissue Engineering and Regenerative Medicine 5, e126 (2011).

15. S. J. An, H. O. Pae, G. S. Oh, B. M. Choi, S. Jeong, S. I. Jang, H. Oh, T. O. Kwon, C. E. Song, and H. T. Chung, International Immunopharmacology 2, 1173 (2002).

16. J. Bixby, T. D. Ray, and F. K. M. Chan, Current Medicinal Chemistry-Anti-Inflammatory and Anti-Allergy Agents 4, 557 (2005).

17. M. Blonska, Z. P. Czuba, and W. Krol, Scandinavian Journal of Immunology 57, 162 (2003).
18. L. C. Denlinger, P. L. Fisette, K. A. Garis, G. Kwon, A. VazquezTorres, A. D. Simon, B. Nguyen, R. A. Proctor, and P. J. Bertics, J. Biol. Chem. 271, 337 (1996).

19. K. Huttunen, M. Ruotsalainen, E. Iivanainen, P. Torkko, M.-L. Katila, and M.-R. Hirvonen, Environ. Toxicol. Pharmacol. 8, 237 (2000).

20. N. I. Moldovan, Antioxidants and Redox Signaling 7, 1409 (2005).

21. R. Olszanecki, A. Gebska, V. I. Kozlovski, and R. J. Gryglewski, J. Physiol. Pharmacol. 53, 571 (2002).

22. K. Sakata, Y. Hirose, Z. Qiao, T. Tanaka, and H. Mori, Cancer Letters 199, 139 (2003).

23. M. S. Cortizo, J. Appl. Polym. Sci. 103, 3785 (2007).

24. M. S. Molinuevo, L. Schurman, A. D. McCarthy, A. M. Cortizo, M. J. Tolosa, M. V. Gangoiti, V. Arnol, and C. Sedlinsky, Journal of Bone and Mineral Research 25, 211 (2010).

25. A. M. Cortizo and S. B. Etcheverry, Mol. Cell Biochem. 145, 97 (1995).

26. M. M. Bradford. Anal. Biochem. 72, 248 (1976).

27. C. A. Grillo, M. A. Reigosa, and M. A. F. L. de Mele, Contraception 81, 343 (2010).

28. T. Okajima, K. Nakamura, H. Zhang, N. Ling, T. Tanabe, T. Yasuda, and R. G, Rosenfeld, Endocrinology 130, 2201 (1992).

29. M. S. Molinuevo, S. B. Etcheverry, and A. M. Cortizo, Toxicology 210, 205 (2005).

30. V. C. Sálice, A. M. Cortizo, C. L. G. Dumm, and S. B. Etcheverry, Mol. Cell. Biochem. 198, 119 (1999).

31. A. M. Cortizo, M. Caporossi, G. Lettieri, and S. B. Etcheverry, Eur. J. Pharmacol. 400, 279 (2000).

32. A. M. Cortizo, G. Ruderman, G. Correa, I. G. Mogilner, and E. J. Tolosa, J. Biomater. Tissue Eng. 2, 125 (2012).

33. E. Velasco-Ortega E, A. Jos, A. M. Cameán, J. Pato-Mourelo, and J. J. Segura-Egea, Mutation Research/Genetic Toxicology and Environmental Mutagenesis 702, 17 (2010).

34. S. Bodhak, S. Bose, and A. Bandyopadhyay, Acta Biomaterialia 5, 2178 (2009).

35. G. Ramírez, S. E. Rodil, H. Arzate, S. Muhl, and J. J. Olaya, Appl. Surf. Sci. 257, 2555 (2011).

36. F. G. Torres, O. P. Troncoso, C. G. Grande, and D. A. Díaz, Materials Science and Engineering: C 31, 1737 (2011).

37. A. P. Marques, R. L. Reis, and J. A. Hunt, Biomaterials 23, 1471 (2002).

38. M. R. Bet MR, G. Goissis, S. Vargas, and H. S. Selistre-de-Araujo, Biomaterials 24, 131 (2003).

39. M. Simon, I. Behrens, L. A. Dailey, M. Wittmar, and T. Kissel, European Journal of Pharmaceutics and Biopharmaceutics 66, 165 (2007).

40. E. M. Harnett, J. Alderman, and T. Wood, Colloids and Surfaces B: Biointerfaces 55, 90 (2007).

41. E. J. P. Jansen, R. E. J. Sladek, H. Bahar, A. Yaffe, M. J. Gijbels, R. Kuijer, S. K. Bulstra, N. A. Guldemond, I. Binderman, and L. H. Koole, Biomaterials 26, 4423 (2005).

42. V. Tangpasuthadol, N. Pongchaisirikul, and V. P. Hoven, Carbohydrate Research 338, 937 (2003).

43. D. F. Williams and S. P. Zhong, International Biodeterioration and Biodegradation 34, 95 (1994).

44. P. Zanchetta and J. Guezennec, Colloids and Surfaces B: Biointerfaces 22, 301 (2001)

45. M. L. B. Palacio, S. R. Schricker, and B. Bhushan, Journal of the Royal Society Interface 8, 630 (2011).

46. T. G. Tihan, M. D. Ionita, R. G. Popescu, and D. Iordachescu, Mater. Chem. Phys. 118, 265 (2009).

47. K. Rezwan, Q. Z. Chen, J. J. Blaker, and A. R. Boccaccini, Biomaterials 27, 3413 (2006).

48. A. J. Salgado, O. P. Coutinho, and R. L., Macromolecular Bioscience 4, 743 (2004) 
49. C. Estrada, A. C. Paz, and L. E. López, Revista EIA 5, 93 (2006).

50. J. Más Estallés, A. Vidaurre, J. M. M. Dueñas, and I. C. Cortázar, The Journal of Materials Science: Materials in Medicine 19, 189 (2008).

51. M. H. Huang, S. Li, and M. Vert, Polymer 45,8675 (2004).

52. E. Bat, T. G. van Kooten, J. Feijen, and D. W. Grijpma, Biomaterials 30,3652 (2009)
53. F. Liu, Z. Zhao, J. Yang, J. Wei, and S. Li, Polym. Degrad. Stab. 94, 227 (2009).

54. H. Yavuz, C. Ceyhun Babac, K. Tuzlakoglu, and E. Piskin, Polym. Degrad. Stab. 75, 431 (2002)

55. J. M. Fernandez, M. S. Cortizo, A. M. Cortizo, and G. A. Abraham, J. Biomater. Tissue Eng. 1, 86 (2011).

Received: $x x$ xxxx xxxx. Accepted: Xx Xxxx xxxx. 